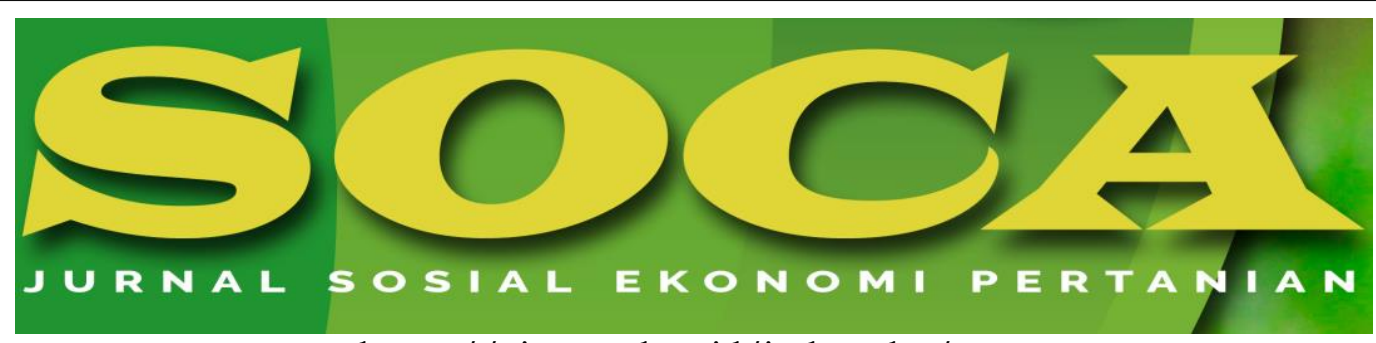

https://ojs.unud.ac.id/index.php/soca

\title{
Farmer Group Institution's Typology and Agricultural Innovation Implementation Sustainability
}

\author{
Nurul Hilmiati \\ Assessment Institute for Agricultural Technology West Nusa Tenggara, Peninjauan Narmada \\ Lombok Barat Street, West Nusa Tenggara 83371 \\ Email: hilmiati@yahoo.com \\ Handphone: 0813373322348
}

Submitted: 27 January 2020; Revised: 9 February 2020; Accepted: 17 February 2020

\begin{tabular}{l}
\hline Keywords: \\
Institutional \\
system \\
development; \\
leadership; \\
transparency; \\
economy \\
activity. \\
\hline
\end{tabular}

Keywords:

Institutional system development; leadership; transparen
economy activity.

\begin{abstract}
Abstrak
Farmer group institutions have been being a target group for various innovations in the agricultural development program. Unfortunately, the aspect of their institutional system is getting ignored frequently. This study aimed to know the farmer group institution performance's determinant factors and its effect on the agricultural innovation implementation sustainability. This was a longitudinal study using a qualitative approach involving ten farmer group institutions (mixed crop-livestock farming) done in Lombok island. The study showed that farmer groups with a good institutional system relatively performed a high level of sustainable innovation implementation in comparison with the group with a poor institutional system. There were four key factors affected the performance and the farmer group institution's achievement: (1) strong leadership; (2) transparency; (3) regular group meeting; (4) and cash generating factor. The study also showed that farmer group institution which didn't have those key factors tended to use the farmer group institution only to complete their physical need (impounded cows for security reason). It rarely uses to empower its group members. These conditions slowly could be developed as an individualistic treat on each group member that prevents the sustainable innovation implementation in the future. The agricultural innovation on this type of group usually only implemented in a short amount of time. Therefore, guidance for a good institutional system in a farmer group institution is required to be conducted to achieve a sustainable and comprehensive agricultural innovation implementation. Some strategies could be used to develop the four key factors to form a good institutional system in the farmer group institution.
\end{abstract}


How to Cite (APA 6th Style):

Hilmiati, N. (2020). Farmer Group Institution 's Typology and Agricultural Innovation Implementation Sustainability. SOCA: Jurnal Sosial Ekonomi Pertanian, 14(2), 205-216.

https://doi.org/https://doi.org/10.24843/SOCA.2020.v14.i02.p02

\section{INTRODUCTION}

Farmer group institutions have been being various national agricultural development programs target. Farmer group institutions are participating as the subject of the program or participants in some agricultural studies. The possible improvement of farmer skill after participating in the program which would be reduced the poverty prevalence in the rural area at the end of the program is the major reason of the selection of the farmer group institution as the target in the national agricultural development program (Dilts, 1999: 36-37). The initial assumption was the farmer group institution leader would able to move their members since the program was only explained specifically to the leader of the group. But, some studies reported that there were some difficulties in disseminating the impact of agricultural programs in a broader agricultural field (Snapp and Heong, 2003, p. 68; Millar, 2009; Millar and Connell, 2010; van de Fliert, et al., 2010).

The majority of the farmer group institutions were only activated during the implementation of the agricultural or an aid program. This type of top-down group approach have criticized to be not effective and efficient because of four main reasons: (1) government limited capacity in reaching some isolated area, (2) top-down type only providing new facilities but not preparing efforts to maintain and sustain the group capability in continuing the implementation of a program, (3) the program was designed by Indonesia's policymakers who possibly had limited knowledge and information about the specific need in some agricultural area and (4) create a dependency cycle because most of the program was designed only to complete its goals but not trying to create a resilient farmer group institutions (Korten, $1983 \mathrm{p}$. 181-183; Hoffmann, Probst, \& Christinck, 2007).

The involvement of the group in the implementation of the agricultural program did not significantly affect the development of the agricultural field, studies which found the effect of group empowerment on the impact of the program and the sustainable agriculture innovation implementation is also still inadequate. This study aimed to know the farmer group institution performance's determinant factors and its effect on the agricultural innovation implementation sustainability.

\section{RESEARCH METHODS}

This was a case study with a purposive sampling technique involving ten farmer group institutions (cow livestock-farming) in Lombok Island. Table 1 shows the farmer group who engaged in this study. The group that participated has been working together with the West Nusa Tenggara Institute of Agricultural Assessment on agriculture technological assessment activities. Agriculture technological assessment could be defined as an adaptive study carried to assist the level of suitability of a technology produced by the Agricultural Research and Development Institute with the physical environment (agriculture-ecosystem), social, culture, and economy in some places to assure the technology performed was specifically appropriate with the location (Indonesia Forestry Minister, 2005). The farmer group institutions that participated in this study were already implemented some 
technology innovation introduced by the West Nusa Tenggara Institute of Agricultural Assessment. During the study, the agriculture technology assessment is already done by the West Nusa Tenggara Institute of Agricultural Assessment. There was one group that participated in this study (Beletak Harapan) that not working together with West Nusa Tenggara Institute of Agricultural Assessment. The agricultural technology information was obtained from another farmer group, namely Ngiring Datu.

The qualitative data collected by a focus group discussion, observation and in-depth interview method (Chambers, 1994; Yin, 2013; Bryman, 2004; Marshall \& Rossman, 2006). The data then tabulated and analyzed thematically (Braun, 2006).

Table 1: Study Location

\begin{tabular}{|c|c|c|c|c|c|c|}
\hline No. & $\begin{array}{c}\text { Farmer } \\
\text { Group } \\
\text { Institution's } \\
\text { Name }\end{array}$ & Village & District & Regency & $\begin{array}{c}\text { Number } \\
\text { of } \\
\text { Member }\end{array}$ & $\begin{array}{c}\text { Cow Cage } \\
\text { Type }\end{array}$ \\
\hline 1 & $\begin{array}{c}\text { Jaya } \\
\text { Gembala }\end{array}$ & Kelebuh & $\begin{array}{l}\text { Praya } \\
\text { Tengah }\end{array}$ & $\begin{array}{l}\text { Middle } \\
\text { Lombok }\end{array}$ & 45 & Colective \\
\hline 2 & $\begin{array}{l}\text { Beriuk Pada } \\
\text { Girang }\end{array}$ & Tandek & & $\begin{array}{l}\text { Middle } \\
\text { Lombok }\end{array}$ & 24 & Colective \\
\hline 3 & $\begin{array}{c}\text { Putri } \\
\text { Bekekem }\end{array}$ & Pringgarata & Pringgarata & $\begin{array}{l}\text { Middle } \\
\text { Lombok }\end{array}$ & 31 & Colective \\
\hline 4 & $\begin{array}{l}\text { Tunggal } \\
\text { Harapan }\end{array}$ & $\begin{array}{l}\text { Tanak } \\
\text { Beak }\end{array}$ & $\begin{array}{c}\text { Batukliang } \\
\text { Utara }\end{array}$ & $\begin{array}{l}\text { Middle } \\
\text { Lombok }\end{array}$ & 31 & Colective \\
\hline 5 & Horsela & $\begin{array}{l}\text { Sembalun } \\
\text { Bumbung }\end{array}$ & Sembalun & $\begin{array}{c}\text { East } \\
\text { Lombok }\end{array}$ & 40 & $\begin{array}{l}\text { Individual/ } \\
\text { Shepherd }\end{array}$ \\
\hline 6 & Ngiring Datu & $\begin{array}{l}\text { Segara } \\
\text { Katon }\end{array}$ & Gangga & $\begin{array}{l}\text { North } \\
\text { Lombok }\end{array}$ & 80 & Colective \\
\hline 7 & $\begin{array}{l}\text { Beletak } \\
\text { Harapan }\end{array}$ & Gangga & Gangga & $\begin{array}{c}\text { North } \\
\text { Lombok }\end{array}$ & 30 & Colective \\
\hline 8 & $\begin{array}{c}\text { Tetu Tanta } \\
\text { Tunaq }\end{array}$ & Sesait & Kayangan & $\begin{array}{l}\text { North } \\
\text { Lombok }\end{array}$ & 52 & $\begin{array}{l}\text { Individual/ } \\
\text { Shepherd }\end{array}$ \\
\hline 9 & $\begin{array}{c}\text { Bina } \\
\text { Keluarga }\end{array}$ & Sesait & Kayangan & $\begin{array}{l}\text { North } \\
\text { Lombok }\end{array}$ & 30 & Colective \\
\hline 10 & $\begin{array}{l}\text { Sambuk } \\
\text { Manis }\end{array}$ & $\begin{array}{l}\text { Beriri } \\
\text { Jarak }\end{array}$ & Wanasab & $\begin{array}{c}\text { East } \\
\text { Lombok }\end{array}$ & 39 & Colective \\
\hline
\end{tabular}

Source : Primary Data (2019)

\section{RESULT AND DISCUSSION}

\section{Innovation Implementation Scope}

In a collaboration with the farmer group institution, West Nusa Tenggara Institute of Agricultural Assessment was introducing a Bali Cattle farming management, especially on the breeding and fattening method. This collaboration was conducted because of the low productivity of its cattle farming. This condition marked by the slow rate of growth, the long interval between breeds ( $\geq 14$ months), a high mortality rate of $>20 \%$, low birth rate as much as $66 \%$ and weaning age that relatively too old (> 6 months). These issues were parallel with some studies that stated the low productivity of cattle farming in West Nusa Tenggara. The limited 
amount of food sources during the dry weather is a major factor that contributed to this condition (Wirdahayati and Bamualim, 1990 cited in Mastika, 2002; Bamualim and Wirdahayati, 2002; Dahlanuddin et al., 2009). The livestock farming system in West Nusa Tenggara characterized by small-scale livestock farming which mainly depends on nature as the food resources and managed by a low-input management system (Talib et al., 2002). This type of farming system is relatively not steady. Farming productivity could be decreased significantly in dry weather because of the limited amount of food and water sources.

There was some livestock farming management innovation offered to improve its productivity. These innovations were defined as integrated herd management strategies that consisted of: (1) mate calendar using a superior variety of the male stud; (2) strategic feeding done by providing quality food to pregnant cows in certain times; (3) calves early weaning; (4) quality food livestock cultivation (using a superior variety of grass, legume plant). Mate calendar innovation is a cattle mating plan in certain months to get the birthing period on a season with sufficient water and food broadly accessible for lactation. Good nutrition would affect the quality of production of the milk, which in the end could affect the growth and immunity of the calves. Strategic feeding is a quality food given by the farmer during the eighth months' pregnancy and two months after pregnancy. In the last two months of pregnancy, the embryo was experiencing a fast period of growth and development. Quality food during this time could increase the weight of the calves and improving female cattle reproduction performance (Bamualim dan Wirdahayati, 2002).

The innovation implementation scope in each farmer group institutions is shown in Table 2. The level of innovation implementation was classified based on the color differences: light green was shown that the innovation sustainably implemented, light yellow was shown that the innovation was only implemented during the study or guidance session, and light brown was shown that the innovation only implemented by some member in the group or not implemented at all. Table 2 shows that based on the proportion in each group, $32.5 \%$ innovation sustainably implemented by the most of the farmer institution group member, $37.5 \%$ sustainably implemented by the minority of the group member or not implemented at all, and $30 \%$ sustainably implemented by the most of the group member but only in a short or specific amount of time. The most type of innovation (light green category) implemented were quality livestock farming cultivation, tactical feeding, and calves early weaning. The hindrances found during the technology implementation were a limitation of agricultural land for livestock farming cultivation, the limited number of superior variety of the cattle male stud, and the farmer's limited knowledge and skill.

Table 2: Technology Implementation Scope and Each Farmer Group Institution Characteristic

\begin{tabular}{|c|c|c|c|c|c|c|c|}
\hline \multirow[b]{2}{*}{ No. } & \multirow{2}{*}{$\begin{array}{c}\text { Farmer } \\
\text { Group } \\
\text { Institutions/ } \\
\text { Performance }\end{array}$} & \multirow[b]{2}{*}{ Innovation } & \multirow[b]{2}{*}{$\begin{array}{c}\text { Technology } \\
\text { Implementation } \\
\text { Scope }\end{array}$} & \multicolumn{4}{|c|}{ Dynamic and Group Characteristic } \\
\hline & & & & Leadership & Tranparency & Meeting & $\begin{array}{l}\text { Income } \\
\text { Source }\end{array}$ \\
\hline 1 & $\begin{array}{l}\text { Jaya } \\
\text { Gembala }\end{array}$ & $\begin{array}{l}\text { Mate } \\
\text { Calendar } \\
\text { with } \\
\text { Superior } \\
\text { Cattle Male } \\
\text { Stud }\end{array}$ & $\begin{array}{lr}\text { After } & \text { the } \\
\text { asesstment } \\
\text { done, the cattle } \\
\text { male stud was } \\
\text { traded. } \\
\text { situation cause } \\
\text { mate calendar } \\
\text { strategy }\end{array}$ & $\begin{array}{l}\text { There was } \\
\text { no leader } \\
\text { replacemen } \\
t \text { for } 10 \\
\text { years } \\
\text { because of } \\
\text { no member } \\
\text { willing to }\end{array}$ & $\begin{array}{l}\text { Transparency } \\
\text { already } \\
\text { implemented, } \\
\text { but group } \\
\text { member still } \\
\text { having } \\
\text { mistrust } \\
\text { feeling about }\end{array}$ & $\begin{array}{l}\text { Group } \\
\text { meeting } \\
\text { conducted } \\
\text { if needed }\end{array}$ & $\begin{array}{l}\text { No } \\
\text { specific } \\
\text { activities } \\
\text { for } \\
\text { group } \\
\text { income }\end{array}$ \\
\hline
\end{tabular}




\begin{tabular}{|c|c|c|c|c|c|c|c|}
\hline \multirow[b]{2}{*}{ No. } & \multirow{2}{*}{$\begin{array}{c}\text { Farmer } \\
\text { Group } \\
\text { Institutions/ } \\
\text { Performance }\end{array}$} & \multirow[b]{2}{*}{ Innovation } & \multirow{2}{*}{$\begin{array}{c}\text { Technology } \\
\text { Implementation } \\
\text { Scope }\end{array}$} & \multicolumn{4}{|c|}{ Dynamic and Group Characteristic } \\
\hline & & & & Leadership & Tranparency & Meeting & $\begin{array}{l}\text { Income } \\
\text { Source }\end{array}$ \\
\hline & & & $\begin{array}{l}\text { couldn't } \\
\text { appropriately } \\
\text { implemented }\end{array}$ & $\begin{array}{l}\text { be the } \\
\text { group } \\
\text { leader }\end{array}$ & $\begin{array}{l}\text { the group } \\
\text { funding } \\
\text { system toward } \\
\text { the leader }\end{array}$ & & \\
\hline & & $\begin{array}{l}\text { Calves } \\
\text { Early } \\
\text { Weaning }\end{array}$ & $\begin{array}{l}\text { The majority of } \\
\text { the member was } \\
\text { implemented } \\
\text { calves early } \\
\text { weaning }\end{array}$ & & & & \\
\hline & & $\begin{array}{l}\text { Superior } \\
\text { Variety } \\
\text { Plant } \\
\text { Cultivation }\end{array}$ & $\begin{array}{l}\text { Implemented but } \\
\text { couldn't } \\
\text { completely done } \\
\text { because of the } \\
\text { limited amoutnt } \\
\text { of food during } \\
\text { the dry season } \\
\text { Implemented, } \\
\text { but couldn't } \\
\text { completely done } \\
\text { because the } \\
\text { limitation of } \\
\text { agricultural area }\end{array}$ & & & & \\
\hline \multirow[t]{2}{*}{2} & $\begin{array}{l}\text { Beriuk Pada } \\
\text { Girang }\end{array}$ & $\begin{array}{l}\text { Mate } \\
\text { Calendar } \\
\text { with } \\
\text { Superior } \\
\text { Cattle Male } \\
\text { Stud }\end{array}$ & $\begin{array}{l}\text { After the } \\
\text { asesstment } \\
\text { done, the cattle } \\
\text { male stud was } \\
\text { traded. This } \\
\text { situation cause } \\
\text { mate calendar } \\
\text { strategy was } \\
\text { couldn't } \\
\text { appropriately } \\
\text { implemented } \\
\text { The majority of } \\
\text { the member was } \\
\text { implemented } \\
\text { calves early } \\
\text { weaning }\end{array}$ & $\begin{array}{l}\text { Weak } \\
\text { leadership, } \\
\text { having no } \\
\text { power in } \\
\text { controlling } \\
\text { the } \\
\text { member }\end{array}$ & $\begin{array}{l}\text { Communicatio } \\
\mathrm{n} \text { rarely } \\
\text { happen } \\
\text { between the } \\
\text { leader and } \\
\text { group member }\end{array}$ & $\begin{array}{l}\text { Group } \\
\text { meeting } \\
\text { conducted } \\
\text { if needed }\end{array}$ & $\begin{array}{l}\text { No } \\
\text { specific } \\
\text { activities } \\
\text { for } \\
\text { group } \\
\text { income }\end{array}$ \\
\hline & & $\begin{array}{l}\text { Superior } \\
\text { Variety } \\
\text { Plant } \\
\text { Cultivation }\end{array}$ & $\begin{array}{l}\text { Implemented but } \\
\text { couldn't } \\
\text { completely done } \\
\text { because of the } \\
\text { limited amoutnt } \\
\text { of food during } \\
\text { the dry season } \\
\text { Implemented, } \\
\text { but couldn't } \\
\text { completely done } \\
\text { because the } \\
\text { limitation of } \\
\text { agricultural area }\end{array}$ & & & & \\
\hline
\end{tabular}




\begin{tabular}{|c|c|c|c|c|c|c|c|}
\hline \multirow[b]{2}{*}{ No. } & \multirow{2}{*}{$\begin{array}{c}\text { Farmer } \\
\text { Group } \\
\text { Institutions/ } \\
\text { Performance }\end{array}$} & \multirow[b]{2}{*}{ Innovation } & \multirow{2}{*}{$\begin{array}{c}\text { Technology } \\
\text { Implementation } \\
\text { Scope }\end{array}$} & \multicolumn{4}{|c|}{ Dynamic and Group Characteristic } \\
\hline & & & & Leadership & Tranparency & Meeting & $\begin{array}{l}\text { Income } \\
\text { Source }\end{array}$ \\
\hline \multirow[t]{2}{*}{3} & $\begin{array}{l}\text { Putri } \\
\text { Bekekem }\end{array}$ & $\begin{array}{l}\text { Mate } \\
\text { Calendar } \\
\text { with } \\
\text { Superior } \\
\text { Cattle Male } \\
\text { Stud } \\
\text { Calves } \\
\text { Early } \\
\text { Weaning }\end{array}$ & $\begin{array}{l}\text { The majority of } \\
\text { the group } \\
\text { member was } \\
\text { implemented } \\
\text { this strategy } \\
\text { The minority of } \\
\text { the group } \\
\text { member was } \\
\text { implemented } \\
\text { this strategy }\end{array}$ & $\begin{array}{l}\text { The leader } \\
\text { was highly } \\
\text { appreciated } \\
\text { by the } \\
\text { group } \\
\text { member, } \\
\text { The village } \\
\text { leader also } \\
\text { highly } \\
\text { motivated } \\
\text { the farmer } \\
\text { group }\end{array}$ & $\begin{array}{l}\text { There was a } \\
\text { routinely } \\
\text { group } \\
\text { financial } \\
\text { report } \\
\text { conducted by } \\
\text { the group }\end{array}$ & $\begin{array}{l}\text { Regular } \\
\text { meeting in } \\
\text { each } \\
\text { month }\end{array}$ & $\begin{array}{l}\text { A } \\
\text { monthly } \\
\text { dues } \\
\text { was } \\
\text { conduct } \\
\text { ed and } \\
\text { shared } \\
\text { to its } \\
\text { member } \\
\text { as El-eid } \\
\text { Mubara } \\
\text { k parcel } \\
\text { in each } \\
\text { year }\end{array}$ \\
\hline & & $\begin{array}{l}\text { Quality } \\
\text { Plant } \\
\text { Cultivation }\end{array}$ & $\begin{array}{l}\text { The majority of } \\
\text { the member } \\
\text { were the planted } \\
\text { superior quality } \\
\text { plant }\end{array}$ & & & & \\
\hline \multirow[t]{3}{*}{4} & $\begin{array}{l}\text { Tunggal } \\
\text { Harapan }\end{array}$ & $\begin{array}{l}\text { Mate } \\
\text { Calendar } \\
\text { with } \\
\text { Superior } \\
\text { Cattle Male } \\
\text { Stud } \\
\text { Calves } \\
\text { Early } \\
\text { Weaning }\end{array}$ & $\begin{array}{l}\text { Did not } \\
\text { implemented }\end{array}$ & $\begin{array}{l}\text { Dominated } \\
\text { by group } \\
\text { leader, but } \\
\text { the } \\
\text { member } \\
\text { relatively } \\
\text { did not } \\
\text { care with } \\
\text { the } \\
\text { decision } \\
\text { taken by } \\
\text { the leader }\end{array}$ & $\begin{array}{l}\text { Group } \\
\text { member still } \\
\text { having } \\
\text { mistrust } \\
\text { feeling about } \\
\text { the group } \\
\text { funding } \\
\text { system toward } \\
\text { the leader. }\end{array}$ & $\begin{array}{l}\text { No group } \\
\text { meeting } \\
\text { conducted }\end{array}$ & $\begin{array}{l}\text { No } \\
\text { specific } \\
\text { activities } \\
\text { for } \\
\text { group } \\
\text { income }\end{array}$ \\
\hline & & $\begin{array}{l}\text { Quality } \\
\text { food for } \\
\text { pregnant } \\
\text { cows }\end{array}$ & $\begin{array}{l}\text { Did not } \\
\text { implemented }\end{array}$ & & & & \\
\hline & & $\begin{array}{l}\text { Quality } \\
\text { Plant } \\
\text { Cultivation }\end{array}$ & $\begin{array}{l}\text { Did not } \\
\text { implemented }\end{array}$ & & & & \\
\hline 5 & Horsela & $\begin{array}{l}\text { Quality } \\
\text { food } \\
\text { (quickstick } \\
\text { / Gliricidia } \\
\text { sepium, } \\
\text { superior } \\
\text { variety of } \\
\text { grass) } \\
\text { Compost } \\
\text { Making } \\
\text { Industry }\end{array}$ & $\begin{array}{l}\text { The minority of } \\
\text { the group } \\
\text { member was } \\
\text { implemented } \\
\text { this strategy }\end{array}$ & $\begin{array}{l}\text { Dominated } \\
\text { by the } \\
\text { group } \\
\text { leader, } \\
\text { Having a } \\
\text { power over } \\
\text { only with } \\
\text { the } \\
\text { member } \\
\text { who have } \\
\text { close } \\
\text { relation }\end{array}$ & $\begin{array}{l}\text { Group } \\
\text { member still } \\
\text { having } \\
\text { mistrust } \\
\text { feeling about } \\
\text { the group } \\
\text { funding } \\
\text { system toward } \\
\text { the leader }\end{array}$ & $\begin{array}{l}\text { Only } \\
\text { conducted } \\
\text { in } \\
\text { asestment } \\
\text { session }\end{array}$ & $\begin{array}{l}\text { No } \\
\text { specific } \\
\text { activities } \\
\text { for } \\
\text { group } \\
\text { income }\end{array}$ \\
\hline
\end{tabular}




\begin{tabular}{|c|c|c|c|c|c|c|c|}
\hline \multirow{3}{*}{ No. } & \multirow{3}{*}{$\begin{array}{c}\text { Farmer } \\
\text { Group } \\
\text { Institutions/ } \\
\text { Performance }\end{array}$} & \multirow[b]{2}{*}{ Innovation } & \multirow{2}{*}{$\begin{array}{c}\text { Technology } \\
\text { Implementation } \\
\text { Scope }\end{array}$} & \multicolumn{4}{|c|}{ Dynamic and Group Characteristic } \\
\hline & & & & Leadership & Tranparency & Meeting & $\begin{array}{l}\text { Income } \\
\text { Source }\end{array}$ \\
\hline & & & $\begin{array}{l}\text { Some group } \\
\text { member was } \\
\text { implemented } \\
\text { this strategy }\end{array}$ & $\begin{array}{l}\text { with the } \\
\text { leader }\end{array}$ & & & \\
\hline
\end{tabular}

\begin{tabular}{|c|c|c|c|c|c|c|c|}
\hline 6 & Ngiring Datu & $\begin{array}{l}\text { Cows } \\
\text { fattening } \\
\text { using } \\
\text { vegetable } \\
\text { hummingbi } \\
\text { rd plant } \\
\text { (Sesbania } \\
\text { grandiflora) } \\
\text { Cultivation } \\
\text { of vegetable } \\
\text { hummingbi } \\
\text { rd plant } \\
\text { (Sesbania } \\
\text { grandiflora) }\end{array}$ & $\begin{array}{l}\text { All member } \\
\text { implemented } \\
\text { innovation } \\
\text { offered. }\end{array}$ & $\begin{array}{l}\text { The leader } \\
\text { was not } \\
\text { really lead } \\
\text { the group } \\
\text { noticeably, } \\
\text { but the } \\
\text { group } \\
\text { secretary is } \\
\text { actively } \\
\text { motivated } \\
\text { and lead } \\
\text { the group }\end{array}$ & $\begin{array}{l}\text { There was a } \\
\text { routinely } \\
\text { group } \\
\text { financial } \\
\text { report }\end{array}$ & $\begin{array}{l}\text { Regular } \\
\text { meeting in } \\
\text { each } \\
\text { month }\end{array}$ & $\begin{array}{l}\text { Compost } \\
\text { making } \\
\text { industry } \\
\text { as group } \\
\text { income } \\
\text { source. }\end{array}$ \\
\hline 7 & $\begin{array}{l}\text { Beletak } \\
\text { Harapan }\end{array}$ & $\begin{array}{l}\text { Cows } \\
\text { fattening } \\
\text { using } \\
\text { vegetable } \\
\text { hummingbi } \\
\text { rd plant } \\
\text { (Sesbania } \\
\text { grandiflora) } \\
\text { Cultivation } \\
\text { of vegetable } \\
\text { hummingbi } \\
\text { rd plant } \\
\text { (Sesbania } \\
\text { grandiflora) }\end{array}$ & $\begin{array}{l}\text { Did not } \\
\text { implemented }\end{array}$ & $\begin{array}{l}\text { Weak } \\
\text { leadership } \\
\text { The leader } \\
\text { was having } \\
\text { no power to } \\
\text { gather the } \\
\text { members } \\
\text { to a group } \\
\text { meeting } \\
\text { Having a } \\
\text { inferior } \\
\text { feeling as a } \\
\text { leader }\end{array}$ & $\begin{array}{l}\text { No } \\
\text { communicatio } \\
\text { n between the } \\
\text { leader and the } \\
\text { group member }\end{array}$ & $\begin{array}{l}\text { No group } \\
\text { meeting } \\
\text { conducted }\end{array}$ & $\begin{array}{l}\text { No } \\
\text { specific } \\
\text { activities } \\
\text { for } \\
\text { group } \\
\text { income }\end{array}$ \\
\hline 8 & $\begin{array}{l}\text { Tetu Tanta } \\
\text { Tunaq }\end{array}$ & $\begin{array}{l}\text { Cows } \\
\text { fattening } \\
\text { using } \\
\text { vegetable } \\
\text { hummingbi } \\
\text { rd plant } \\
\text { (Sesbania } \\
\text { grandiflora) } \\
\text { Cultivation } \\
\text { of vegetable } \\
\text { hummingbi } \\
\text { rd plant } \\
\text { (Sesbania } \\
\text { grandiflora) }\end{array}$ & $\begin{array}{l}\text { The vegetable } \\
\text { hummingbird } \\
\text { cultivation only } \\
\text { conducted by a } \\
\text { few farmer } \\
\text { because the } \\
\text { limited suppy of } \\
\text { its seed. }\end{array}$ & $\begin{array}{l}\text { Leader was } \\
\text { actively } \\
\text { participate } \\
\text { d and tried } \\
\text { to involve } \\
\text { the } \\
\text { member in } \\
\text { group } \\
\text { activities. }\end{array}$ & $\begin{array}{l}\text { The financial } \\
\text { situation of } \\
\text { the group } \\
\text { routinely } \\
\text { reported to } \\
\text { the member. }\end{array}$ & $\begin{array}{l}\text { Regular } \\
\text { meeting in } \\
\text { each } \\
\text { month }\end{array}$ & $\begin{array}{l}\text { Using } \\
\text { monthly } \\
\text { dues as } \\
\text { the cash } \\
\text { generati } \\
\text { ng group } \\
\text { activity } \\
\text { and } \\
\text { conduct } \\
\text { ed a } \\
\text { saving } \\
\text { and } \\
\text { loans } \\
\text { group } \\
\text { for the } \\
\text { member }\end{array}$ \\
\hline
\end{tabular}




\begin{tabular}{|c|c|c|c|c|c|c|c|}
\hline \multirow[b]{2}{*}{ No. } & \multirow{2}{*}{$\begin{array}{c}\text { Farmer } \\
\text { Group } \\
\text { Institutions/ } \\
\text { Performance }\end{array}$} & \multirow[b]{2}{*}{ Innovation } & \multirow{2}{*}{$\begin{array}{c}\text { Technology } \\
\text { Implementation } \\
\text { Scope }\end{array}$} & \multicolumn{4}{|c|}{ Dynamic and Group Characteristic } \\
\hline & & & & Leadership & Tranparency & Meeting & $\begin{array}{l}\text { Income } \\
\text { Source }\end{array}$ \\
\hline 9 & $\begin{array}{l}\text { Bina } \\
\text { Keluarga }\end{array}$ & $\begin{array}{l}\text { Cows } \\
\text { fattening } \\
\text { using } \\
\text { vegetable } \\
\text { hummingbi } \\
\text { rd plant } \\
\text { (Sesbania } \\
\text { grandiflora) } \\
\text { Cultivation } \\
\text { of vegetable } \\
\text { hummingbi } \\
\text { rd plant } \\
\text { (Sesbania } \\
\text { grandiflora) }\end{array}$ & $\begin{array}{l}\text { Did not } \\
\text { implemented }\end{array}$ & $\begin{array}{l}\text { There was } \\
\text { mistrust } \\
\text { feeling } \\
\text { between } \\
\text { the group } \\
\text { member, }\end{array}$ & $\begin{array}{l}\text { No financial } \\
\text { group report } \\
\text { conducted by } \\
\text { this group }\end{array}$ & $\begin{array}{l}\text { No group } \\
\text { meeting } \\
\text { scheduled }\end{array}$ & $\begin{array}{l}\text { No } \\
\text { specific } \\
\text { activities } \\
\text { for } \\
\text { group } \\
\text { income }\end{array}$ \\
\hline \multirow[t]{4}{*}{10} & $\begin{array}{l}\text { Sambuk } \\
\text { Manis }\end{array}$ & $\begin{array}{l}\text { Quality } \\
\text { Plant } \\
\text { Cultivation }\end{array}$ & $\begin{array}{l}\text { The majority of } \\
\text { group member } \\
\text { was highly } \\
\text { participated in } \\
\text { implementing } \\
\text { this strategy }\end{array}$ & $\begin{array}{l}\text { The leader } \\
\text { was } \\
\text { actively } \\
\text { lead the } \\
\text { group and } \\
\text { highly } \\
\text { apreciated } \\
\text { by the } \\
\text { member }\end{array}$ & $\begin{array}{l}\text { Routinely } \\
\text { financial } \\
\text { report is } \\
\text { conducted in } \\
\text { each month }\end{array}$ & $\begin{array}{l}\text { Regular } \\
\text { meeting in } \\
\text { each } \\
\text { month }\end{array}$ & $\begin{array}{l}\text { Compost } \\
\text { making } \\
\text { industry } \\
\text { as group } \\
\text { income } \\
\text { source. }\end{array}$ \\
\hline & & $\begin{array}{l}\text { Flushing } \\
\text { food for } \\
\text { pregnant } \\
\text { cows }\end{array}$ & $\begin{array}{l}\text { The majority of } \\
\text { group member } \\
\text { was highly } \\
\text { participated in } \\
\text { implementing } \\
\text { this strategy }\end{array}$ & & & & \\
\hline & & $\begin{array}{l}\text { Calves } \\
\text { Early } \\
\text { Weaning }\end{array}$ & $\begin{array}{l}\text { Calves early } \\
\text { weaning was } \\
\text { implemented by } \\
\text { some livestock } \\
\text { farmer }\end{array}$ & & & & \\
\hline & & $\begin{array}{l}\text { Compost } \\
\text { Making } \\
\text { Industry }\end{array}$ & $\begin{array}{l}\text { The compost } \\
\text { making industry } \\
\text { was conducted } \\
\text { but the cash } \\
\text { generating } \\
\text { activity through } \\
\text { this strategy was } \\
\text { not effective } \\
\text { because the lack } \\
\text { of the compost } \\
\text { purchaser. }\end{array}$ & & & & \\
\hline
\end{tabular}

\section{Farmer Group Institution Characteristic and Technology Implementation}

This study was able to identify the characteristics and farmer group institution performance with its level of innovation adoption which is shown in Table 2. The performance of each farmer group institution was described by colors as shown in the table: the light green showed a good level, light yellow showed a low level, and light brown showed a very low level.

Based on the result shows in Table 2, there was a correlation between group performance and sustainable innovation adoption. Some farmer group institution with a good group performance such as Putri Bekekem and Ngiring Datu was implemented the agricultural innovation sustainably during and after the guidance session. Other farmer groups with a low and very low group performance such as 
Jaya Gembala, Beriuk Pada Girang, Tunggal harapan, Horsela, Beletak Harapan, and Bina Keluarga did not perform the agricultural innovation sustainably. A farmer group namely Tetu Tanta Tunak was having a good group performance, but only a few members implemented the cultivation of vegetable hummingbird plants (Sesbania grandiflora). This happened due to the limited amount of its seed. This was parallel with a study done by Hilmiati et al. (2016) that found sustainable innovation required some supporting elements to be implemented: awareness, knowledge and skill improvement, and access to the innovation element.

Results also showed that four determinant key factors play an important role in building a good institutional system in a farmer group institutional system: strong leadership, transparency, regular meeting, and cash-generating activity. Strong leadership is being the main factor and an important key in a good institutional system in a group. Farmer institutional group with the weak figure of leader usually could not be organized dan lead its members to achieve the group goal. Some farmer groups with weak leadership in this study were Beriuk in Girang, Tunggal Harapan, Beletak Harapan, and Bina Keluarga. Farmers in this type of group usually stay as members because of security reasons (collective impounded cow system). They were only physically attached to the leader and the group but did not emotionally attach with the group which is poor for the group institutional system development.

Transparency is the second factor affected a good institutional group system. Based on the in-depth interview conducted in this study, some key members in the Ngiring Datu farmer group institution stated that transparency plays an important role in group harmony. A group meeting was regularly conducted in each month to report the financial group situation. While the opposite situation has happened in the Tunggal Harapan farmer group institution. During the FGD session, some group members were stated their opinion about some policies made by the leader of the group. Some members also stated their suspicious feeling toward the group leader about the group fund condition. Quarry and Ramirez (2009, p. 21) and Servaes \& Malikhao (2008, p. 170) stated that transparency and trust are a foundation of communication in creating a conducive atmosphere on information and knowledge exchange in a group. Some groups with transparency issues such as Tunggal Harapan and Bina Keluarga were having a low level of technology adoption.

Another key factor in maintaining a good institutional factor in a farmer group institution is a regular meeting. Murdah, Ngiring Datu group secretary stated that regular meeting is important to facilitate the need of the member in expressing their opinion about ongoing or planned group activities and evaluating all group activities. The regular meeting also providing chances to clarify the group funding which able to decrease the mistrust feeling of the group member. Information and the need for new technology adoption also easier to disseminate through these regular meetings. This opinion was similar with some studies (Chambers, 1994b; Millar and Connell, 2010; Petheream, 2000) that found member contribution in a group could contribute to a higher commitment and sense of ownership in a group which able to affect the implementation of innovation offered through a group approach. Quarry and Ramirez (2009, p. 20) stated that interpersonal dialogue able to create discussion about some issues and the solution for those issues between members. This study also showed that farmer group institution with a low level of performance was not having a regular meeting and a low level of technology adoption. Some members were also having mistrust feeling toward the group leader due to no chance available in discussing and reporting the group funding system. The mistrust issues were very dangerous for group harmony. Mistrust caused apathetic traits in the members in 
their group activity, which also affected the innovation adoption disseminated through group approach.

The last determinant factor in building a good institutional system in a group is the cash-generating factor. Leader of Putri Bekekem and secretary of the Ngiring Datu farmer institution group stated that group income was playing an important role in their group operational activity. These farmer groups were having a compost home industry as their group cash-generating activity. The profit from the compost home industry was shared with its members in the form of member's savings and loans. The profit also would be shared at the end of the year. Similar to the Ngiring Datu farmer group, the Putri Bekekem farmer group is also conducting a cashgenerating activity by collecting in monthly dues to the group. The accumulation of the monthly dues would be shared with the member at each year in a form of Eid alFitr parcel. This activity is good in maintaining member's emotional attachment to the group.

\section{CONCLUSION}

A good institutional system in a farmer group institution is an important key to create a sustainable impact on the agricultural field. Supporting technological innovation adoption able to improve crop productivity and also would increase farmer's income and wellness in the future. This study concluded that there were four determinants key factors in building a good institutional system in a farmer group institution: (1) strong leadership to move the group member and group dynamic; (2) transparency to minimalize the friction on the group; (3) regular group meeting that provides a chance for self-reflection and discussed things that required on improving the group performance; (4) and productive group activities which able to motivate and strengthen the bond between the group and the member.

\section{RECOMMENDATION}

Agricultural studies and programs are required in disseminating technology and innovation on the farmer group institution to improve their crop productivity. Unfortunately, the implementation of these activities only highlighting the physical aspects, the innovation itself and the livestock as its agricultural object. Contrary to these implementations, non-physical factors also closely related to the sustainability of the program, one of those factors is institutional factors. Therefore, to create a more extensive range and sustainable agricultural implementation program, supervision needs to be conducted to build a good group institutional system. The institutional system could be classified into two major elements: (1) human resources empowerment which targeted the farmer as the group member and (2) group empowerment which targeted the farmer group institution whose goal is to reach the farmer wellness. 


\section{REFERENCES}

Bamualim A; Wirdahayati RB. 2002. Nutrition and management strategies to improve Bali cattle productivity in Nusa Tenggara. Proceedings of the ACIAR workshop Strategies to improve Bali cattle in Indonesa, Bali, Indonesia, 4-7 February 2002. p. 17-22.

Braun V, Clarke V. 2006. Using Thematic Analysis In Psychology. Qualitative Research in Psychology. 3(2): 77-101.

Bryman A. 2004. Social Research Methods. Second Edition. Oxford: Oxford Univeristy Press.

Chambers R. 1994. The Origin And Practice Of Participatory Rural Appraisal. World Development. 22(7): 953-969.

Dahlanuddin; Muzani A; Sutaryono YA; McDonald C. 2009. Strategi peningkatan produktivitas sapi Bali pada sistem kandang kompleks: pengalaman di Lombok Tengah, NTB. Paper presented at the Pengembangan Sapi Bali Berkelanjutan dalam Sistem Peternakan Rakyat, Mataram.

Dilts R. 1990. Researchers from The Village: An Indonesian Non-Government Rural Action And Community Development Program. Innotech Journal. 1: 34-42.

Hilmiati N, Sutartha, Panjaitan T. 2016. Managing Sustainable Practice Changes In A Low Input Bali Cattle Production System In West Sumbawa. International Journal of Sustainable Development. 9(1): 47-53.

Hoffmann V, Probst K, Christinck A. 2007. Farmers And Researchers: How Can Collaborative Advantages Be Created In Participatory Research And Technology Development? Agriculture and Human Values. 24(3): 355-368.

Korten F. 1983. Community Participation: A Management Perspective On Obstacles And Pptions. In David C. Korten and Felipe B. Alfonso (eds.) Bureaucracy and the Poor: Closing the Gap. Singapore: McGraw-Hill International Book Company. p. 181-200.

Mastika, I. M. 2002. Feeding strategies to improve the production performance and meat quality of Bali cattle (Bos sondaicus). ACIAR Proceedings, Strategies to Improve Bali Cattle in Eastern Indonesia. pp. 1013.

Millar J. 2009. Adapting Extension Approaches to Cultural Environments In South East Asia: Experiences Form Laos And Indonesia. Extension Farming Systems Journal. 5(1):143-148.

Millar J, Connell, J. 2010. Strategies for Scaling Out Impacts From Agricultural Systems Change: The Case Of Forages And Livestock Production in Laos. Agricultural Human Values. 27:213-225.

Ministry of Agriculture. 2005. Ministry of Agriculture Decree Number03/PERMENTAN/2005. Jakarta

Petheram RJ. 2000. Introduction And Review. In JR Petheram (Ed.) A Manual Of Tools For Participatory R\&D In Dryland Cropping Areas (p. 1-15). Creswick: Rural Industries Research\&Development Corporation. 
Quarry W, Ramirez R. 2009. Communication For Another Development: Listening Before Telling. London: Zed Books.

Servaes J, Malikhao P. 2008. Development Communication: Approaches In An International Perspective. In J Servaes (Ed.), Communication for Development and Social Change (2 ed.). New Delhi: SAGE India.

Snapp S, Heong KL. 2003. Scaling Up And Out. In Pound P, Snapp S, McDougall C,. Braun AR (Eds.), Managing Natural Resources For Sustainable Livelihoods: Uniting Science, Participation (p. 67-83). London: Earthscan.

Talib, C., Entwistle, K., Siregar, A., Budiarti-Turner, S., Lindsay, D. 2002. Survey of population and production dynamics of Bali cattle and existing breeding programs in Indonesia. ACIAR Proceedings, Strategies to Improve Bali Cattle in Eastern Indonesia. pp. 3-9.

Van de Fliert E, Budi C, Hendayana R, Murray-Prior R. 2010. Pilot Roll-Out: Adaptive Research In Farmer's World. Extension Farming Systems Journal. 6(1): 63-71.

Yin RK. 2003. Case study research: design and methods. Third ed. Vol. 5. London: SAGE Publications. 